PTH reverses radiation-induced hypocellularity during distraction osteogenesis

\title{
Role of Parathyroid Hormone in Regeneration of Irradiated Bone in a Murine Model of Mandibular Distraction Osteogenesis
}

Stephen Y. Kang M.D. ${ }^{1,3}$, Sagar S. Deshpande B.S. ${ }^{1}$, Alexander R. Zheutlin B.S. ${ }^{1}$, Alexis Donneys M.D. ${ }^{1}$, Jose J. Rodriguez M.D. ${ }^{1}$, Noah S. Nelson B.S. ${ }^{1}$, Peter A. Felice M.D. ${ }^{1}$, Douglas B. Chepeha M.D. M.S.P.H. ${ }^{4}$, Steven R. Buchman M. $D^{1,2}$

1. Craniofacial Research Laboratory, University of Michigan Medical School, Ann Arbor, MI

2. Section of Plastic Surgery, University of Michigan Health System, Ann Arbor, MI

3. Department of Otolaryngology-Head and Neck Surgery, The Ohio State University Wexner Medical Center, Columbus, $\mathrm{OH}$

4. Department of Otolaryngology-Head and Neck Surgery, University of Toronto, Toronto, ON

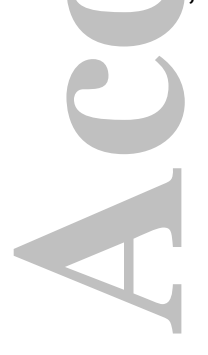

This work is supported both by a grant from the National Institute of Health (NIHR01 CA 125187-01) to Steven R. Buchman and by a research grant "Effect of This is the author manuscript accepted for publication and has undergone full peer review but has not been through the copyediting, typesetting, pagination and proofreading process, which may lead to differences between this version and the Version record. Please cite this article as doi:10.1002/ hed.24612. 
PTH reverses radiation-induced hypocellularity during distraction osteogenesis

Anabolic PTH on Distracted Mandibular Bone" from the AAO-HNS to Douglas B.

Chepeha. The authors have no commercial associations or financial disclosures.

This work was presented as an oral presentation at the 2014 American Society of Plastic Surgeons meeting in Chicago, IL.

\section{Corresponding Author:}

Steven R. Buchman, M.D.

University of Michigan Health System

Pediatric Plastic Surgery Section

4-730 C.S. Mott Children's Hospital

1540 E. Hospital Drive

Ann Arbor, Ml 48109-4215

sbuchman@med.umich.edu

Running Title: PTH reverses radiation-induced hypocellularity during distraction osteogenesis

Keywords: Parathyroid hormone, distraction osteogenesis, radiation, mandible, union

John Wiley \& Sons, Inc.

This article is protected by copyright. All rights reserved. 
PTH reverses radiation-induced hypocellularity during distraction osteogenesis

Background: Our aim was to measure the histologic and histomorphometric effects of PTH treatment on irradiated bone undergoing distraction osteogenesis (DO).

Methods: Thirty-four rats were divided into three groups. The control group underwent $\mathrm{DO}$ and the radiation control group underwent radiation treatment (RT) prior to DO. The PTH group underwent RT and received PTH during DO. Quantitative histology and histomorphometry was performed.

Results: RT resulted in a depletion of osteocytes and increase in empty lacunae. Treatment with PTH resulted in an increase in osteocyte counts and decrease in empty lacunae $(p<0.05)$, restoring osteocytes to levels seen in nonradiated bone $(p=0.121)$. RT decreased bone-volume-to-tissue-volume ratio and increased osteoid-volume-to-tissue-volume (OV/TV) ratio, signifying increased immature bone formation. PTH treatment restored OV/TV ratio to that observed in non-radiated bone.

Conclusions: PTH treatment of irradiated bone enhanced bone regeneration and restored osteocyte counts and OV/TV ratio to levels comparable to nonradiated bone. 
PTH reverses radiation-induced hypocellularity during distraction osteogenesis
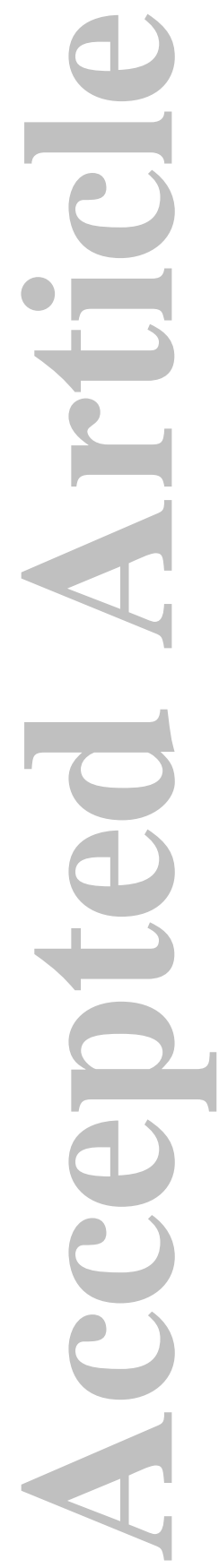

John Wiley \& Sons, Inc.

This article is protected by copyright. All rights reserved. 
PTH reverses radiation-induced hypocellularity during distraction osteogenesis

\section{Introduction}

Radiation treatment is a critical component of the multidisciplinary management of head and neck cancer. However, radiation treatment also severely damages surrounding tissue, impairs wound healing, and degrades bone within the treatment field ${ }^{1-3}$. Radiation treatment delivered to bone has been shown to cause immediate and delayed osteocyte death ${ }^{1,2,4}$, decrease bone mineral density ${ }^{5}$, decrease biomechanical strength ${ }^{6}$, and impair osseous vascularity on a macroscopic and microscopic level ${ }^{6-9}$. The most severe form of osseous damage from radiation treatment occurs in the form of osteoradionecrosis $^{210}$, which remains a challenging clinical entity.

Adjunctive agents that enhance osseous healing in the irradiated field could be quite beneficial. Parathyroid hormone (PTH) has emerged as a potential therapeutic agent as it has been shown to promote bone anabolism and is FDA approved in the treatment of osteoporosis ${ }^{11}$. However, while PTH has shown tremendous anabolic potential in patients with osteoporosis, more data are needed to determine if PTH can enhance osseous regeneration within irradiated bone. If PTH were to enhance bone regeneration within an irradiated field, it could have tremendous therapeutic potential for clinical translation to patients with advanced oral cavity cancers requiring osseous free tissue transfer and adjuvant radiation treatment, as well as patients with osteoradionecrosis. In this study, we employ a murine model of distraction osteogenesis in order to study the histologic and histomorphometric effects of radiation treatment and intermittent PTH on the regenerate bone. We hypothesize that radiation 
PTH reverses radiation-induced hypocellularity during distraction osteogenesis

treatment will result in decreased osteocyte levels, increased empty lacunae, and replacement of mature, mineralized bone with immature, osteoid formation. We further hypothesize that treating irradiated bone with intermittent PTH will reverse these radiation-induced effects, restoring histological and histomorphometric architecture similar to that of non-radiated bone.

\section{Materials and Methods}

\section{Experimental Groups}

Thirty-four twelve-week-old isogenic male Lewis rats were randomly assigned to one of three experimental groups: DO, xDO, and xDO-PTH (Figure 1). All groups underwent surgical placement of mandibular distractors and underwent mandibular distraction osteogenesis. The $D O(n=12)$ group was the normal control group and underwent mandibular distraction osteogenesis. The DO group did not receive radiation treatment. The $\mathrm{xDO}(\mathrm{n}=10)$ group was the radiation control group and received preoperative radiation treatment followed by mandibular distraction osteogenesis. The xDO-PTH $(n=12)$ group received preoperative radiation treatment followed by mandibular distraction osteogenesis and intermittent subcutaneous PTH (60 $\mu \mathrm{g} / \mathrm{kg}$, once daily) beginning on the first day of distraction for a total duration of 21 days. Our animal protocol has been reviewed and approved by the University Committee on the Use and Care of Animals. The experimental timeline is shown in Figure 2.

\section{Radiation Treatment}

Rats were acclimated for 7 days in light and temperature controlled 
PTH reverses radiation-induced hypocellularity during distraction osteogenesis

facilities and given hard chow and water without restriction. Radiation was delivered to the $\mathrm{xDO}$ and $\mathrm{xDO}-\mathrm{PTH}$ groups; the DO group did not receive radiation. Prior to radiation, rats were anesthetized with inhaled isoflurane. Induction was begun at $4 \%$, after which anesthesia was maintained at $1.5 \%$.

All radiation treatments were performed in the Irradiation Core at the University of Michigan Cancer Center using a Philips R250 orthovoltage unit (250kV, 15 mA; Kimtron Medical, Woodbury, CT). Radiation was delivered to the left hemimandible, 2-mm posterior to the third molar. Lead shielding was used to ensure localized delivery and to protect surrounding tissue. 7 Gy was delivered daily for 5 days for a total fractionated treatment dose of $35 \mathrm{~Gy}$, which is the bioequivalent dose of $70 \mathrm{~Gy}$ in humans [30]. Dosimetry was carried out using an ionization chamber connected to an electrometer system, which was directly traceable to a National Institute of Standards and Technology calibration.

After completion of radiation, the XDO-PTH and XDO groups were allowed to recover for 14 days. During this period, all three groups were acclimated to a soft chow high-calorie diet (Hills-Columbus Serum; Columbus, Ohio). The percent of ration of calcium and phosphorus were $0.95 \%$ and $1.05 \%$ respectively, and the content of vitamin D was $4.5 \mathrm{IU}$ per gram.

\section{Surgery and Postoperative Care}

All three groups underwent surgical placement of a mandibular distraction device with unilateral left mandibular osteotomy. The details of the surgical procedure have been previously described in detail ${ }^{12}$. The surgery was 
PTH reverses radiation-induced hypocellularity during distraction osteogenesis

performed 14 days after completion of radiation in the xDO-PTH and xDO groups. Anesthesia was achieved with inhalational isoflurane (4\% induction, $1.5 \%$ maintenance) and subcutaneous buprenorphine $(0.3 \mathrm{mg} / \mathrm{kg})$. A single dose of subcutaneous gentamicin $(5 \mathrm{mg} / \mathrm{kg})$ was given preoperatively.

Postoperatively, rats were given two doses of subcutaneous gentamicin ( 5 $\mathrm{mg} / \mathrm{kg})$ every 12 hours to prevent infection. Subcutaneous buprenorphine $(0.03$ $\mathrm{mg} / \mathrm{kg}$ ) was given every twelve hours through postoperative day (POD) 5 .

Staples were removed at POD 10. Weights were measured daily and subcutaneous lactated ringer solution was delivered as needed. Pin care was provided with Silvadene (Monarch Pharmaceuticals, Inc., Bristol, TN). Maxillary incisors were clipped weekly because of overgrowth from crossbite.

\section{Mandibular Distraction Osteogenesis and Region of Interest}

r.

All rats began left mandibular distraction osteogenesis on POD 4.

Distraction was performed at a rate of $0.3 \mathrm{~mm}$ every 12 hours to a total distraction gap of $5.1 \mathrm{~mm}$. The $5.1 \mathrm{~mm}$ distraction gap was chosen because it is a critical size defect in the murine mandible and was previously shown to be reliably distracted with complete bony union ${ }^{12}$. The final distraction was completed on POD 12. Subjects then underwent a 28-day consolidation period prior to sacrifice and histological analysis.

\section{Parathyroid Hormone}

Intermittent recombinant human PTH (1-34) (Bachem, CA) was 
PTH reverses radiation-induced hypocellularity during distraction osteogenesis

administered to the $\mathrm{xDO}-\mathrm{PTH}$ group in the postoperative period, beginning on the first day of distraction (POD 4). PTH (60 $\mu \mathrm{g} / \mathrm{kg}$ ) was delivered in intermittent fashion, once daily, and was injected subcutaneously over the right hindlimb. Treatment duration was 3 weeks; a total of 21 doses were delivered and treatment was completed on POD 25. The PTH treatment dose and duration employed in this study has previously been shown to improve vascularity, union quality, bone mineral density, and bone volume fraction in a similar murine model of $\mathrm{DO}^{5,9}$.

\section{Tissue Harvest}

Specimens were processed and embedded following a protocol previously described ${ }^{13}$. Bone formed within the $5.1 \mathrm{~mm}$ distraction gap was isolated and selected as our region of interest (ROI) for histologic analysis. Coronal sections of the ROI were performed at a thickness of $7 \mu \mathrm{m}$, using a Leica Reichert-Jung 2030 Biocut Microtome (Leica, Wetzlar, Germany). After the sections were mounted on glass slides, every seventh slide was stained with Gomoro one-step trichome. Two slides representative of the mid gap of the ROI were selected per specimen for histologic analysis.

\section{Quantitative Histomorphometry}

\section{Digital color analysis}

Using the imaging analysis software program Bioquant NOVA Osteo version 7 (R\&M Biometrics, Nashville, TN), digital images of each slide were obtained for the placement of a standard template over the ROI and color thresholding. The digital color analysis thresholds a blue-green color to the 
PTH reverses radiation-induced hypocellularity during distraction osteogenesis

mature, mineralized bone color, and a red-pink color to the osteoid, or immature bone. Two images for each slide are then created, one image where mature, mineralized bone is replaced by red overlay, and another image where osteoid is replaced by red overlay. Three independent reviewers, who were blinded with respect to the treatment groups, reviewed these images and obtained the

\section{following:}

- Tissue volume, representing the total volume of the ROI template.

- Bone volume, representing the volume of mineralized bone within the ROI.

- Osteoid volume, representing the volume of nonmineralized, immature bone and vacuoles within the ROI.

Bone volume to tissue volume and osteoid volume to tissue volume ratios were calculated using the Bioquant software.

\section{Union After Distraction Osteogenesis}

Union of the regenerate region was determined by three blinded and independent reviewers. The presence of a union was defined as a solid bony bridging, in addition to an absence of motion across the distraction osteogenesis gap.

\section{Point Counting of Osteocytes and Empty Lacunae}

Using the Bioquant software, the ROI was superimposed onto the digital image. Nine high-power-field images at 16x magnification were randomly selected and stored as Tagged Image File Format files. Point counting of osteocyte and empty lacunae was performed by three independent reviewers, 
PTH reverses radiation-induced hypocellularity during distraction osteogenesis who were blinded with respect to the treatment groups. Osteocytes were single cells that resided within lacunae.

\section{Statistical Analysis}

Statistical analysis was performed using SPSS Statistics software V. 20 (IBM; Armonk, NY). The data were compared using one-way analysis of variance (ANOVA). Post-hoc analysis was performed by either Tukey or GamesHowell method, depending on the homogeneity of variances. Significance was assigned as $p<0.05$.

\section{Results}

\section{Union After Distraction Osteogenesis}

Union rate in the DO, xDO and xDO-PTH groups are shown in Figure 3.

The non-radiated control group (DO group) exhibited complete union in all specimens $(100 \%)$. In contrast, the union rate in the group that received radiation treatment (xDO group) was considerably reduced, as only $43 \%$ of these mandibles formed a complete union. However, the addition of PTH to irradiated bone (XDO-PTH) improved the union rate to $80 \%$ (Figure 3 ).

\section{Quantitative histologic analysis}

Figure 4 shows representative histologic samples from the non-radiated group (DO group), radiation treated group (xDO group), and the radiation treated group receiving intermittent PTH (xDO-PTH group). Compared to the DO group, 
PTH reverses radiation-induced hypocellularity during distraction osteogenesis

radiation treatment resulted in a significant decrease in osteocyte count and increase in empty lacunae. The group receiving radiation treatment and $\mathrm{PTH}$ (xDO-PTH) showed osteocyte counts that were restored to non-radiated levels. Empty lacunae were significantly decreased in the xDO-PTH group compared to the $\mathrm{xDO}$ group, although empty lacunae were not decreased to non-radiated levels (Figures 4 and 5).

In all quantitative metrics, a significant difference was identified between treatment groups as determined by one-way ANOVA $(p<0.05)$. As expected, radiation treatment group $(x D O)$ resulted in a significant depletion of osteocytes and a significant increase in empty lacunae when compared to the non-radiated control group (DO). Specifically, compared to the DO group, the xDO group showed a greater than 2-fold decrease in osteocyte count after radiation treatment $(67.9$ v. $139.3, p<0.001)$. The xDO group showed a greater than 5 -fold increase in empty lacunae after radiation treatment, compared to the nonradiated DO group (12.3 v. 2.36, $\mathrm{p}=0.001)$ (Figure 5).

Treatment of radiated bone with intermittent $\mathrm{PTH}$ resulted in significant restoration of osseous cellularity, resulting in reversal of radiation-induced hypocellularity in the xDO-PTH group (radiation treatment followed by distraction and intermittent PTH). Specifically, compared to the non-PTH treated radiation group (XDO), treatment with PTH resulted in a 1.84-fold increase in osteocyte count (125.0 v. $67.9, p<0.001)$. Furthermore, compared to the xDO group, the xDO-PTH group had a 2.6-fold decrease in empty lacunae after PTH treatment (4.67 v. 12.3, $p=0.005)$ (Figure 5). 
PTH reverses radiation-induced hypocellularity during distraction osteogenesis Intermittent treatment with PTH after radiation treatment in the XDO-PTH group resulted in a significant restoration of osteocytes to levels observed in the non-radiated DO group. There were no measurable differences in osteocyte count between non-radiated and distracted bone (DO group) and bone undergoing radiation treatment and intermittent PTH treatment (xDO-PTH group). Specifically, there was no significant difference in osteocyte count between the non-radiated DO group and the group undergoing radiation treatment followed by intermittent PTH (xDO-PTH) (139.3 v. 125.0, $p=0.121$ ) (Figure 5). While treatment with PTH significantly decreased the number of empty lacunae compared to the radiation control group, treatment with PTH did not restore empty lacunae levels to non-radiated levels (4.67 v. $2.36, p<0.05)$.

\section{Histomorphometry}

Compared to the DO group, radiation treatment in the $\mathrm{XDO}$ group resulted in a significant decrease in mature bone formation as determined by histomorphometry. Representative histomorphometric samples from the DO, $\mathrm{xDO}$, and $\mathrm{xDO}-\mathrm{PTH}$ groups are shown in Figure 6. Specifically, compared to the DO group, radiation treatment in the XDO group resulted in a 1.6-fold decrease in bone volume to tissue volume ratio (BV/TV), $(0.331 \mathrm{v} .0 .516, p=0.008)$. The $x D O$ group also showed an increase in immature bone formation as determined by osteoid volume to tissue volume ratio (OV/TV). Specifically, compared to the DO group, radiation treatment resulted in a 1.7-fold increase in immature bone formation as measured by OV/TV (0.257 v. 0.149, $p=0.024)$ (Figure 7). 
PTH reverses radiation-induced hypocellularity during distraction osteogenesis

Compared to the $\mathrm{xDO}$ group, treatment with $\mathrm{PTH}$ was able to increase the bone volume to tissue volume ratio in the $\mathrm{xDO}-\mathrm{PTH}$ group, but this difference was not significant $(0.4171$ v. $0.3483, p=0.420)$. Similarly, treatment with PTH decreased immature bone formation as determined by osteoid volume to tissue volume ratio, but this difference was also not significant $(0.1837$ v. 0.2572 , $\mathrm{p}=0.178)$ (Figure 7).

While radiation treatment resulted in a significant increase of immature bone formation as determined by osteoid volume to tissue volume ratio in the xDO and DO groups, treatment with $\mathrm{PTH}$ lessened this radiation-induced effect. Specifically, in comparison to the non-radiated DO group, the xDO-PTH group showed no significant difference in OV/TV (1.837 v. 1.491, p=0.345). However, while PTH treatment was able to reduce the effect of radiation on bone volume loss, it was unable to completely reverse the BV/TV ratio to non-radiated levels. Specifically, compared to the DO group, the xDO-PTH group showed a significant decrease in BV/TV ratio (0.4171 v. 0.516, $p=0.026)$ (Figure 7).

\section{Discussion}

Radiation treatment has been shown to result in poorer regenerate outcomes in mandibular distraction osteogenesis ${ }^{5,9,14-16}$, and this study confirmed that radiation treatment results in a significant depletion of osseous cellularity in a murine model of distraction osteogenesis. However, treatment of radiated bone with intermittent $\mathrm{PTH}$ reversed radiation induced hypocellularity. In fact, PTH restored osteocyte counts in irradiated bone to baseline levels, as 
PTH reverses radiation-induced hypocellularity during distraction osteogenesis

there was no significant difference in osteocyte count between the irradiated group that received PTH (xDO-PTH group) and the group that underwent distraction alone, without radiation treatment (DO group). Treatment with PTH also increased the degree of mature bone formation within the region of interest, although this was not restored to normal control levels. Previous work has shown that PTH improves biomechanical strength ${ }^{6}$, union quality, bone mineral density ${ }^{5}$, and osseous vascularity ${ }^{9}$ in irradiated bone. This is the first report to demonstrate the ability of PTH to increase mature bone formation and restore osteocyte count to non-radiated levels in bone.

While the complete cellular mechanism by which PTH promotes bone anabolism remains to be elucidated, PTH has been shown to have a pleiotropic anabolic effect on bone formation ${ }^{17}$, increasing osteoblast number by promoting osteoblastogenesis, inhibiting osteoblast apoptosis, and reactivating lining cells to resume matrix synthesizing function ${ }^{17,18}$. Radiation treatment specifically reduces proliferation of vasculature and osteoblasts ${ }^{19}$. While more studies are needed to determine the mechanism by which PTH ameliorates the effects of radiation treatment, the findings of this study clearly demonstrate the ability of PTH to preserve and restore osteocyte counts in irradiated bone to levels that are comparable to non-radiated bone.

Teriparatide, also known as recombinant human PTH (1-34), is an attractive adjunctive agent to study because it is already in clinical use and a U.S. Food and Drug Administration-approved therapy for osteoporosis ${ }^{11,20,21}$. With regard to potential therapeutic use for osseous wounds in the head and 
PTH reverses radiation-induced hypocellularity during distraction osteogenesis

neck, Bashutski et al. ${ }^{22}$ used intermittent PTH to accelerate alveolar bone regeneration in the oral cavity after periodontal surgery, evidenced by a significant radiographic improvement in linear resolution of osseous defects as well as a reduction in probing depth. Case reports suggest that PTH may have a role in treating bisphosphonate-related osteonecrosis of the jaw ${ }^{14,23,24}$. These findings suggest tremendous therapeutic potential as an adjunctive treatment to complex wounds in the head and neck, where osseous wound healing is of paramount importance to functional outcome.

However, one large clinical hurdle exists, as a history of radiation treatment delivered to bone is currently a contraindication to PTH treatment ${ }^{25,26}$. This contraindication exists due to the observation of osteosarcoma in rats chronically treated with $\mathrm{PTH}{ }^{27}$. However, the animals in that particular study were treated at higher doses than those used in human studies, and for time spans up to 2 years, representing $80-90 \%$ of their normal time span, complicating comparisons to clinical trials that use short-term use of PTH. The same authors studied PTH-related osteosarcoma in monkeys treated with daily PTH, and reported zero cases of osteosarcoma ${ }^{28}$ suggesting that this effect may be species-dependent and not applicable to human subjects ${ }^{26}$. Harper et al. reported one case of osteosarcoma among $>300,000$ patients worldwide that have been treated with teriparatide $(\mathrm{PTH})^{24}$. As a result, causality between teriparatide and osteosarcoma in humans has yet to be established. However, the safety of teriparatide use in patients with a history of head and neck cancer and/or radiation treatment delivered to the mandible remains to be determined. 
PTH reverses radiation-induced hypocellularity during distraction osteogenesis

This study adds to a growing literature demonstrating that PTH has tremendous potential to enhance osseous regeneration in irradiated bone. PTH has previously been shown to enhance biomechanical strength, bone mineral density, and union formation in irradiated bone ${ }^{5,6}$. This histologic and histomorphometric study adds new data, demonstrating the ability of PTH to reverse radiation induced hypocellularity and enhance mature bone formation in a murine model of distraction osteogenesis, and further supports the potential use of PTH to enhance osseous regeneration in the irradiated mandible.
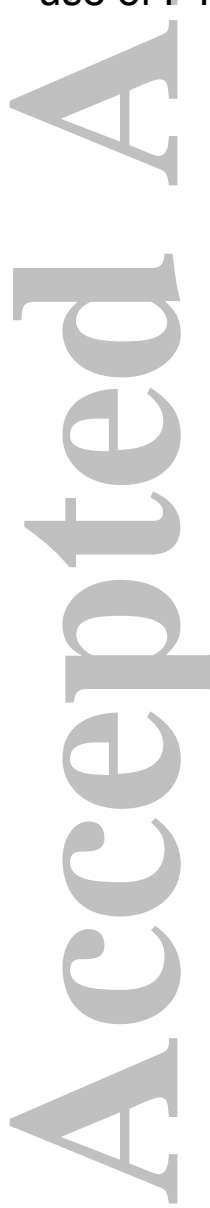
PTH reverses radiation-induced hypocellularity during distraction osteogenesis

\section{References}

1. Costantino PD, Friedman CD, Steinberg MJ. Irradiated bone and its management. Otolaryngol Clin North Am. 1995;28(5):1021-1038.

2. Marx RE. Osteoradionecrosis: a new concept of its pathophysiology. J Oral Maxillofac Surg. 1983;41(5):283-288.

3. Teknos TN, Myers LL. Surgical reconstruction after chemotherapy or radiation. Problems and solutions. Hematol Oncol Clin North Am. 1999;13(4):679-687.

4. Sanger JR, Matloub HS, Yousif NJ, Larson DL. Management of osteoradionecrosis of the mandible. Clin Plast Surg. 1993;20(3):517-530.

5. Gallagher KK, Deshpande S, Tchanque-Fossuo CN, et al. Role of parathyroid hormone therapy in reversing radiation-induced nonunion and normalization of radiomorphometrics in a murine mandibular model of distraction osteogenesis. Head Neck. 2013;35(12):1732-1737.

6. Deshpande SS, Gallagher KK, Donneys A, et al. Parathyroid hormone therapy mollifies radiation-induced biomechanical degradation in murine distraction osteogenesis. Plast Reconstr Surg. 2013;132(1):91e-100e.

7. Cutright DE, Brady JM. Long-term effects of radiation on the vascularity of rat bone--quantitative measurements with a new technique. Radiat Res. 1971;48(2):402-408.

8. Deshpande SS, Gallagher KK, Donneys A, et al. Stem cells rejuvenate radiation-impaired vasculogenesis in murine distraction osteogenesis. Plast Reconstr Surg. 2015;135(3):799-806.

9. Kang SY, Deshpande SS, Donneys A, et al. Parathyroid hormone reverses radiation induced hypovascularity in a murine model of distraction osteogenesis. Bone. 2013;56(1):9-15.

10. Teng MS, Futran ND. Osteoradionecrosis of the mandible. Curr Opin Otolaryngol Head Neck Surg. 2005;13(4):217-221.

11. Neer RM, Arnaud CD, Zanchetta JR, et al. Effect of parathyroid hormone (1-34) on fractures and bone mineral density in postmenopausal women with osteoporosis. N Engl J Med. 2001;344(19):1434-1441.

12. Buchman SR, Ignelzi MA, Jr., Radu C, et al. Unique rodent model of distraction osteogenesis of the mandible. Ann Plast Surg. 2002;49(5):511519. 
PTH reverses radiation-induced hypocellularity during distraction osteogenesis

13. Tchanque-Fossuo CN, Monson LA, Farberg AS, et al. Dose-response effect of human equivalent radiation in the murine mandible: part I. A histomorphometric assessment. Plast Reconstr Surg. 2011;128(1):114121.

14. Fregene A, Jing XL, Monson LA, Buchman SR. Alteration in volumetric bone mineralization density gradation patterns in mandibular distraction osteogenesis following radiation therapy. Plast Reconstr Surg. 2009;124(4):1237-1244.

15. Inyang AF, Schwarz DA, Jamali AM, Buchman SR. Quantitative histomorphometric assessment of regenerate cellularity and bone quality in mandibular distraction osteogenesis after radiation therapy. J Craniofac Surg. 2010;21(5):1438-1442.

16. Schwarz DA, Jamali AM, Kakwan MS, Fregene A, Arman KG, Buchman $\mathrm{SR}$. Biomechanical assessment of regenerate integrity in irradiated mandibular distraction osteogenesis. Plast Reconstr Surg. 2009;123(2 Suppl):114S-122S.

17. Jilka RL. Molecular and cellular mechanisms of the anabolic effect of intermittent PTH. Bone. 2007;40(6):1434-1446.

18. Kim SW, Pajevic PD, Selig M, et al. Intermittent parathyroid hormone administration converts quiescent lining cells to active osteoblasts. J Bone Miner Res. 2012;27(10):2075-2084.

19. Willey JS, Lloyd SA, Robbins ME, et al. Early increase in osteoclast number in mice after whole-body irradiation with $2 \mathrm{~Gy} X$ rays. Radiat Res. 2008;170(3):388-392.

20. Black DM, Greenspan SL, Ensrud KE, et al. The effects of parathyroid hormone and alendronate alone or in combination in postmenopausal osteoporosis. N Engl J Med. 2003;349(13):1207-1215.

21. Finkelstein JS, Hayes A, Hunzelman JL, Wyland JJ, Lee H, Neer RM. The effects of parathyroid hormone, alendronate, or both in men with osteoporosis. N Engl J Med. 2003;349(13):1216-1226.

22. Bashutski JD, Eber RM, Kinney JS, et al. Teriparatide and osseous regeneration in the oral cavity. N Engl J Med. 2010;363(25):2396-2405.

23. Cheung A, Seeman E. Teriparatide therapy for alendronate-associated osteonecrosis of the jaw. N Engl J Med. 2010;363(25):2473-2474.

24. Harper KD, Krege JH, Marcus R, Mitlak BH. Osteosarcoma and teriparatide? J Bone Miner Res. 2007;22(2):334. 
PTH reverses radiation-induced hypocellularity during distraction osteogenesis

25. Girotra M, Rubin MR, Bilezikian JP. The use of parathyroid hormone in the treatment of osteoporosis. Rev Endocr Metab Disord. 2006;7(1-2):113121.

26. Pleiner-Duxneuner J, Zwettler E, Paschalis E, Roschger P, NellDuxneuner V, Klaushofer K. Treatment of osteoporosis with parathyroid hormone and teriparatide. Calcif Tissue Int. 2009;84(3):159-170.

27. Vahle JL, Sato M, Long GG, et al. Skeletal changes in rats given daily subcutaneous injections of recombinant human parathyroid hormone (134) for 2 years and relevance to human safety. Toxicol Pathol. 2002;30(3):312-321.

28. Vahle JL, Zuehlke U, Schmidt A, Westmore M, Chen P, Sato M. Lack of bone neoplasms and persistence of bone efficacy in cynomolgus macaques after long-term treatment with teriparatide [rhPTH(1-34)]. J Bone Miner Res. 2008;23(12):2033-2039.

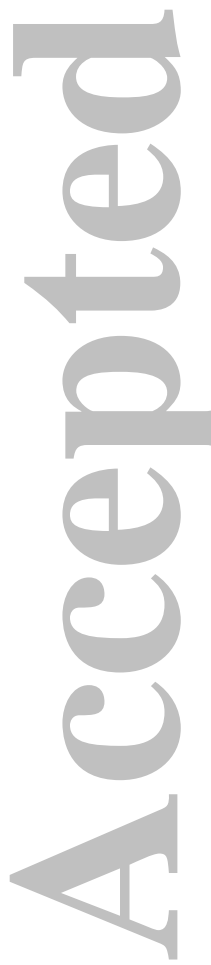


PTH reverses radiation-induced hypocellularity during distraction osteogenesis

Figure 1. Experimental groups. The DO group underwent mandibular distraction alone, without radiation treatment. The xDO group underwent radiation treatment followed by mandibular distraction. The xDO-PTH group underwent radiation treatment, mandibular distraction, and intermittent PTH treatment for 21 days. DO, distraction osteogenesis; Gy, gray; PTH, parathyroid hormone.

(1)

Figure 2. Experimental timeline. In the xDO-PTH group, intermittent subcutaneous parathyroid hormone $(\mathrm{PTH})$ treatment was given daily for 21 days beginning on the first day of mandibular distraction. XRT, radiation treatment; DO, distraction osteogenesis; PTH, parathyroid hormone.

Figure 3. Union rate in the $\mathrm{DO}, \mathrm{xDO}$, and $\mathrm{xDO}-\mathrm{PTH}$ groups. Union rate was ascertained based on clinical assessment of the regenerate for bony bridging without motion in the distraction osteogenesis gap. The DO (no radiation treatment) group had a $100 \%$ bony union rate, while the xDO (radiation treatment) group only formed a bony union in $43 \%$ of specimens. The xDO-PTH group (radiation treatment $+\mathrm{PTH}$ ) formed complete unions in $80 \%$ of specimens.

Figure 4. Representative sections of formation during distraction osteogenesis in non-radiated bone (DO), irradiated bone (XDO), and irradiated bone treated with PTH (XDO-PTH). Specimens were stained with Gomori trichrome and are shown at $16 x$ magnification. Radiation treatment in the xDO group resulted in a 
PTH reverses radiation-induced hypocellularity during distraction osteogenesis

significant depletion of osteocytes and increase in empty lacunae. However, treatment of irradiated bone with PTH (xDO-PTH) resulted in a significant increase in osteocyte count and decrease in empty lacunae. There was no significant difference in osteocyte count in non-radiated bone (DO) and irradiated bone treated with PTH (xDO-PTH). The black arrow shows an osteocyte residing within a lacunae and the red arrow shows an empty lacunae.

Figure 5. Quantitative histologic analysis. Radiation treatment resulted in a significant depletion of osteocytes in the xDO group when compared to nonradiated group $(\mathrm{DO})(\mathrm{p}<0.001)$. Treatment of irradiated bone with $\mathrm{PTH}$ resulted in a significant increase osteocyte counts $(p<0.001)$. In fact, no significant difference in osteocyte count existed between the non-radiated group (DO) and the irradiated group treated with PTH $(x D O-P T H)(p=0.121) . \quad Y$ axis represents osteocytes per high power field. With regard to empty lacunae, radiation treatment in the $\mathrm{xDO}$ group resulted in a significant increase in empty lacunae compared to non-radiated bone $(p=0.001)$. Treatment with PTH in the $x D O-P T H$ group resulted in a significant decrease in empty lacunae $(p=0.005)$, albeit not to levels seen in non-radiated bone. $\mathrm{Y}$ axis represents empty lacunae per high power field.

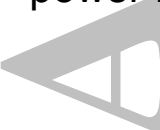

Figure 6. Representative digital images used for histomorphometric assessment obtained by the imaging analysis software program Bioquant NOVA Osteo version 7 (R\&M Biometrics, Nashville, TN) to quantitatively assess the ratio of 
PTH reverses radiation-induced hypocellularity during distraction osteogenesis

mature, mineralized bone formation to immature osteoid formation within the region of interest. In the Standard column, mature, mineralized bone is represented by turquoise while osteoid is represented in pink-purple. In the Bone Volume column, red overlay represents mature mineralized bone formation. In Osteoid Volume column, red overlay represents immature, osteoid formation. These representative images show that compared to non-radiated bone (DO group), radiation treatment (xDO group) results in decreased mature bone formation and greater osteoid formation. Treatment with PTH (xDO-PTH group) attenuates the effect of radiation treatment, resulting in greater areas of mature, mineralized bone formation, and less osteoid formation in irradiated bone undergoing distraction osteogenesis (DO). When treated with PTH, the pattern of bone formation in irradiated bone undergoing DO is strikingly similar to nonradiated bone.

radia

Figure 7. Quantitative histomorphometric analysis. Compared to the nonradiated DO group, radiation treatment in the $\mathrm{XDO}$ group resulted in a significant decrease in bone-volume-to-total-volume ratio $(B V / T V)(p=0.008)$. Treatment of irradiated bone with $\mathrm{PTH}$ resulted in an increase in BV/TV, but this difference was not significant $(p=0.420)$. Radiation treatment resulted in a significantly greater osteoid-volume-to-total-volume (OV/TV) in the xDO group, compared to the non-radiated DO group $(p=0.024)$. Treatment of irradiated bone with PTH resulted in a decrease in OV/TV, but this difference was not significant $(p=0.178)$. 
PTH reverses radiation-induced hypocellularity during distraction osteogenesis

However, there was no detectable difference in OV/TV between the non-radiated DO group and the irradiated group treated with PTH $(p=0.345)$.

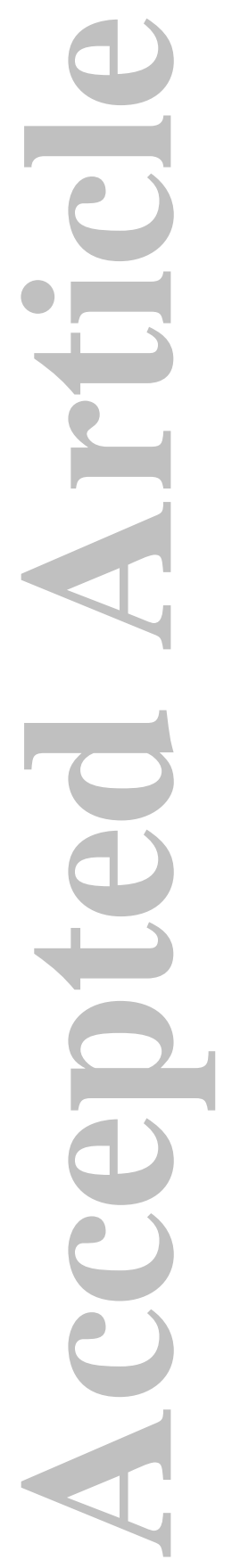

John Wiley \& Sons, Inc.

This article is protected by copyright. All rights reserved. 


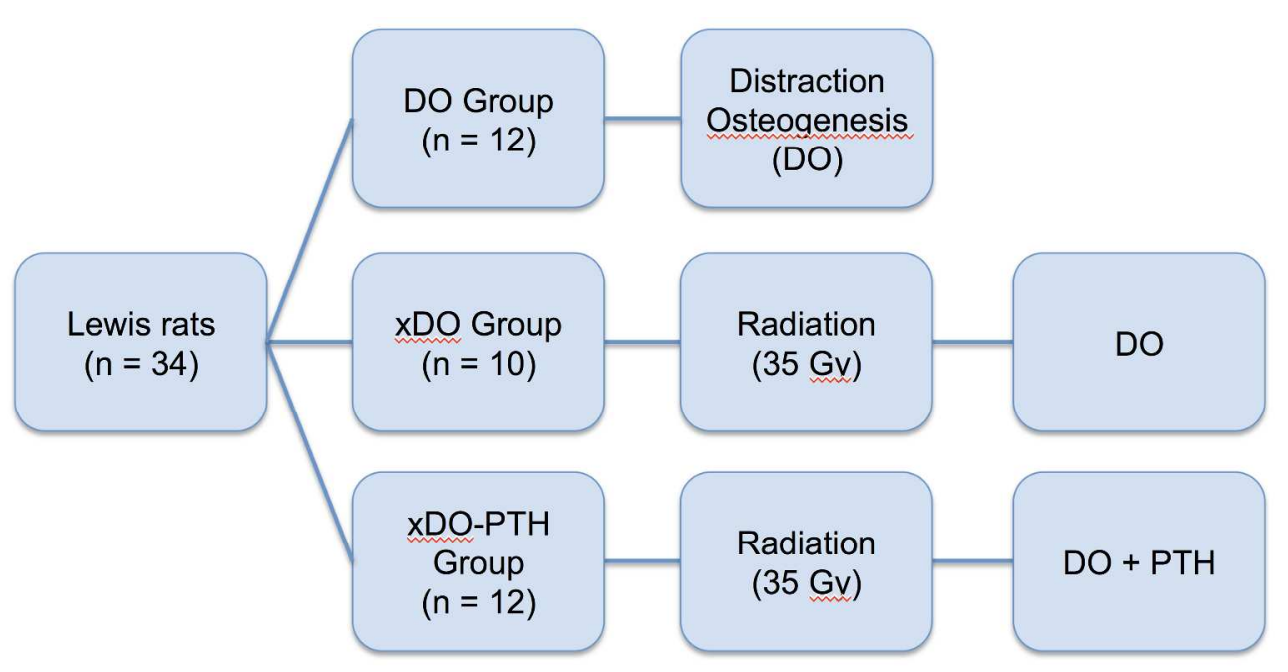

Figure 1. Experimental groups. The DO group underwent mandibular distraction alone, without radiation treatment. The XDO group underwent radiation treatment followed by mandibular distraction. The XDOPTH group underwent radiation treatment, mandibular distraction, and intermittent PTH treatment for 21 days. DO, distraction osteogenesis; Gy, gray; PTH, parathyroid hormone.

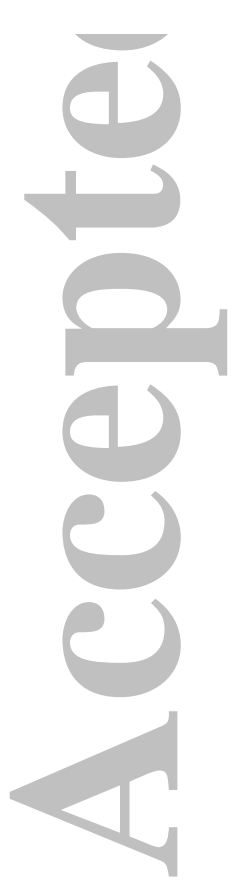

$642 \times 324 \mathrm{~mm}(144 \times 144$ DPI $)$

John Wiley \& Sons, Inc.

This article is protected by copyright. All rights reserved. 


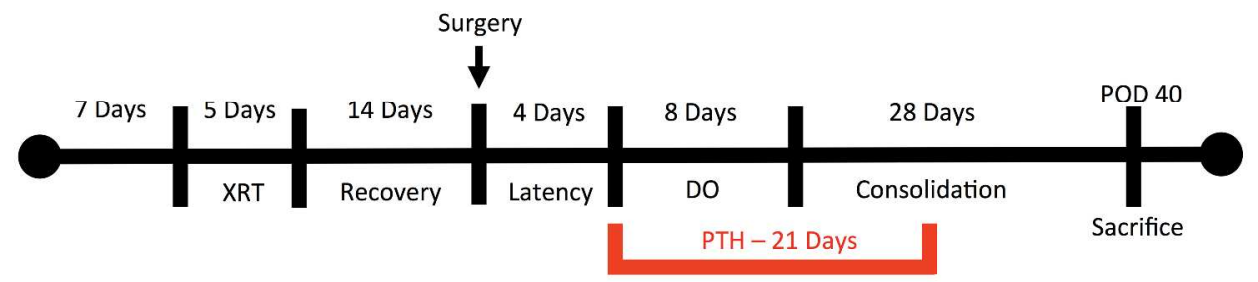

Figure 2. Experimental timeline. In the XDO-PTH group, intermittent subcutaneous parathyroid hormone (PTH) treatment was given daily for 21 days beginning on the first day of mandibular distraction. XRT, radiation treatment; DO, distraction osteogenesis; PTH, parathyroid hormone.

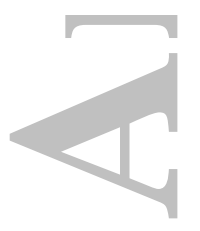

$773 \times 186 \mathrm{~mm}(144 \times 144 \mathrm{DPI})$

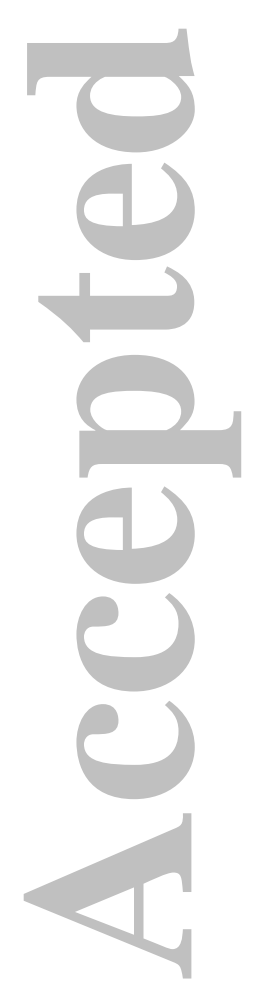

John Wiley \& Sons, Inc.

This article is protected by copyright. All rights reserved. 


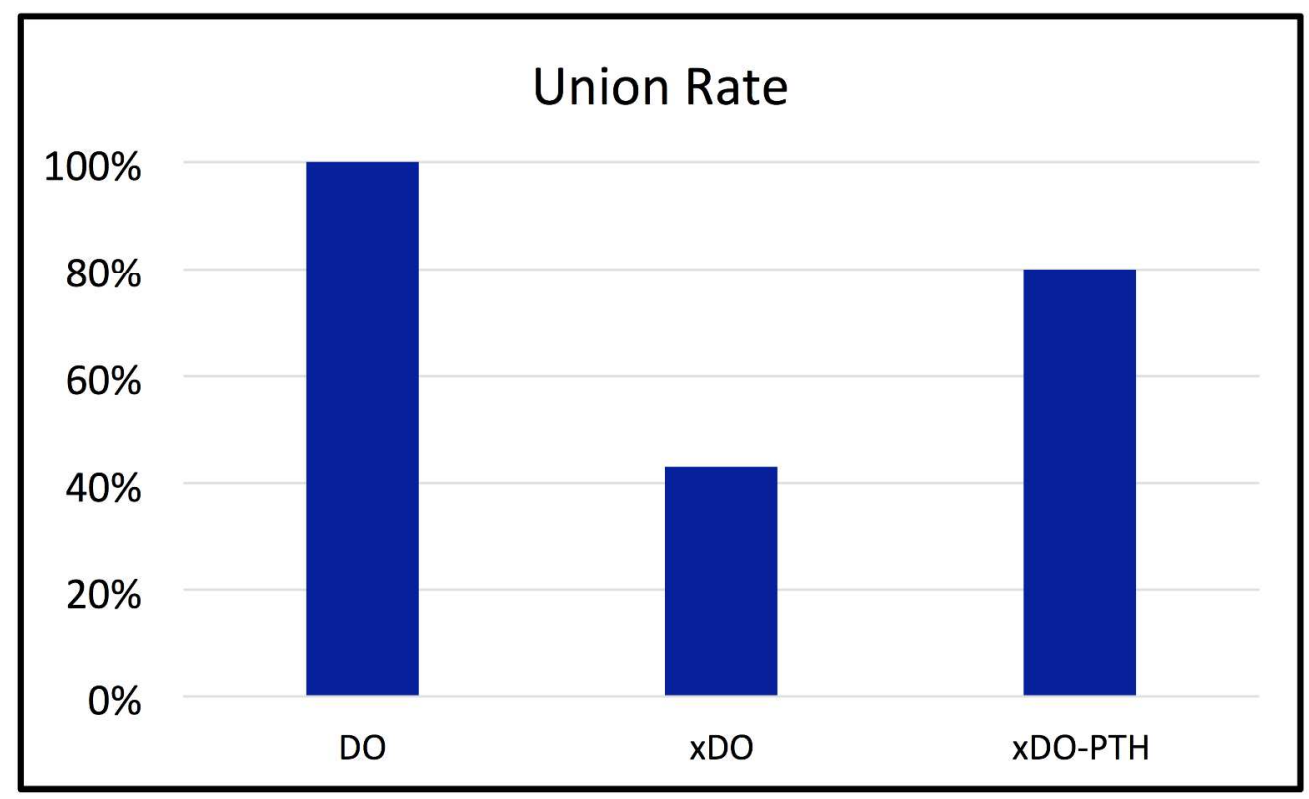

Figure 3. Union rate in the DO, xDO, and xDO-PTH groups. Union rate was ascertained based on clinical assessment of the regenerate for bony bridging without motion in the distraction osteogenesis gap. The DO (no radiation treatment) group had a $100 \%$ bony union rate, while the xDO (radiation treatment) group only formed a bony union in $43 \%$ of specimens. The xDO-PTH group (radiation treatment + PTH) formed complete unions in $80 \%$ of specimens.

$516 \times 311 \mathrm{~mm}(144 \times 144$ DPI $)$

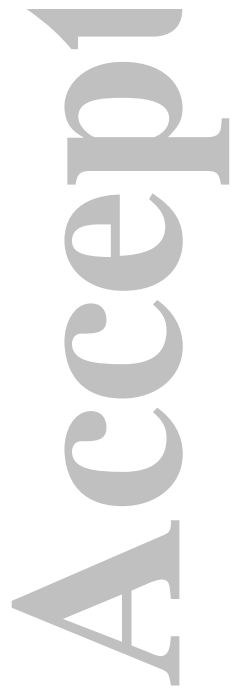

John Wiley \& Sons, Inc.

This article is protected by copyright. All rights reserved. 

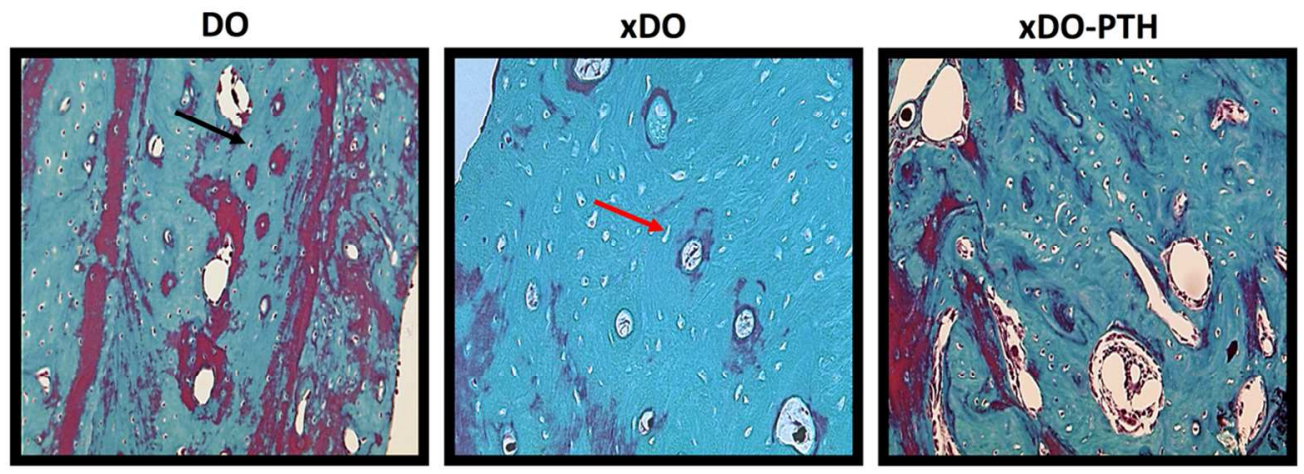

Figure 4. Representative sections of formation during distraction osteogenesis in non-radiated bone (DO), irradiated bone (xDO), and irradiated bone treated with PTH (XDO-PTH). Specimens were stained with Gomori trichrome and are shown at 16x magnification. Radiation treatment in the xDO group resulted in a significant depletion of osteocytes and increase in empty lacunae. However, treatment of irradiated bone with PTH (XDO-PTH) resulted in a significant increase in osteocyte count and decrease in empty lacunae. There was no significant difference in osteocyte count in non-radiated bone (DO) and irradiated bone treated with PTH (XDO-PTH). The black arrow shows an osteocyte residing within a lacunae and the red arrow shows an empty lacunae.

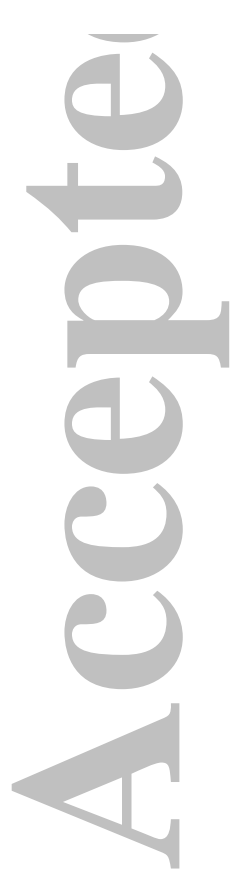

$108 \times 43 \mathrm{~mm}(300 \times 300 \mathrm{DPI})$

John Wiley \& Sons, Inc.

This article is protected by copyright. All rights reserved. 


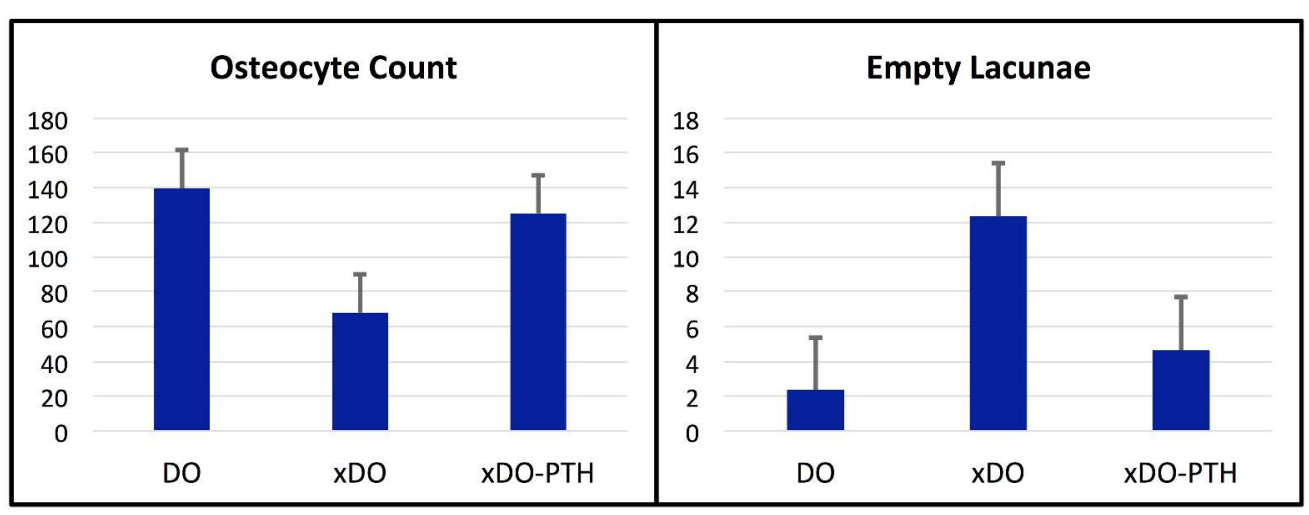

Figure 5. Quantitative histologic analysis. Radiation treatment resulted in a significant depletion of osteocytes in the XDO group when compared to non-radiated group (DO) $(p<0.001)$. Treatment of irradiated bone with PTH resulted in a significant increase osteocyte counts $(p<0.001)$. In fact, no significant difference in osteocyte count existed between the non-radiated group (DO) and the irradiated group treated with PTH (xDO-PTH) $(p=0.121)$. Y-axis represents osteocytes per high power field. With regard to empty lacunae, radiation treatment in the xDO group resulted in a significant increase in empty lacunae compared to non-radiated bone $(p=0.001)$. Treatment with PTH in the XDO-PTH group resulted in a significant decrease in empty lacunae $(p=0.005)$, albeit not to levels seen in non-radiated bone. $Y$-axis represents empty lacunae per high power field.

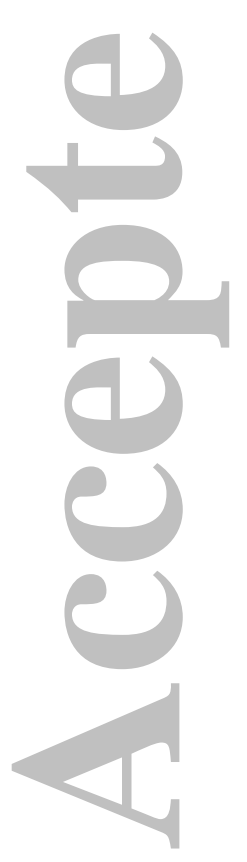

$802 \times 308 \mathrm{~mm}(144 \times 144 \mathrm{DPI})$

John Wiley \& Sons, Inc.

This article is protected by copyright. All rights reserved. 


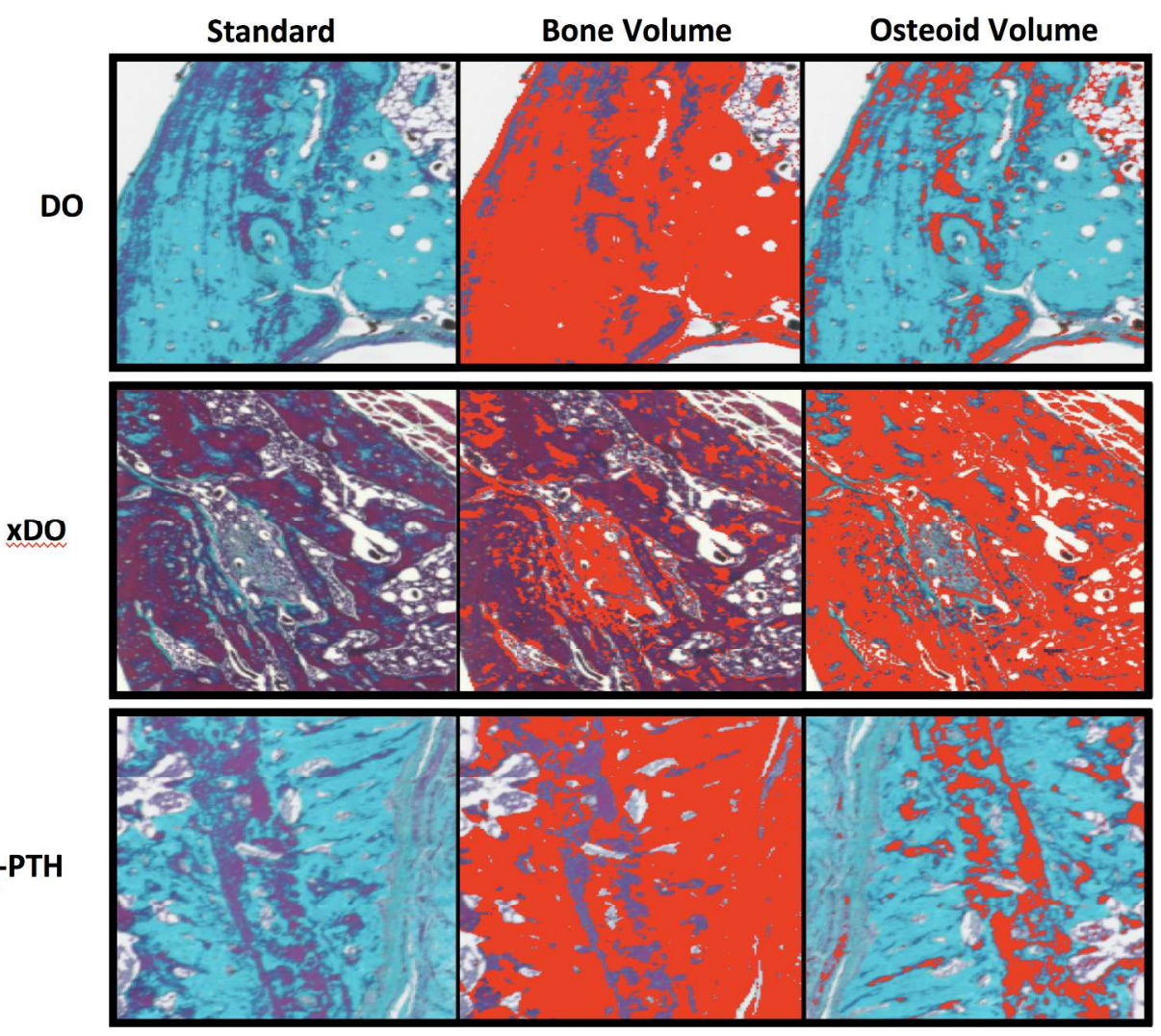

Figure 6. Representative digital images used for histomorphometric assessment obtained by the imaging analysis software program Bioquant NOVA Osteo version 7 (R\&M Biometrics, Nashville, TN) to quantitatively assess the ratio of mature, mineralized bone formation to immature osteoid formation within the region of interest. In the Standard column, mature, mineralized bone is represented by turquoise while osteoid is represented in pink-purple. In the Bone Volume column, red overlay represents mature mineralized bone

formation. In Osteoid Volume column, red overlay represents immature, osteoid formation. These representative images show that compared to non-radiated bone (DO group), radiation treatment (XDO group) results in decreased mature bone formation and greater osteoid formation. Treatment with PTH

(xDO-PTH group) attenuates the effect of radiation treatment, resulting in greater areas of mature, mineralized bone formation, and less osteoid formation in irradiated bone undergoing distraction osteogenesis (DO). When treated with PTH, the pattern of bone formation in irradiated bone undergoing DO is strikingly similar to non-radiated bone.

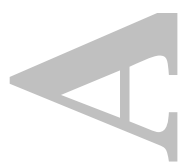

$518 \times 421 \mathrm{~mm}(144 \times 144 \mathrm{DPI})$ 


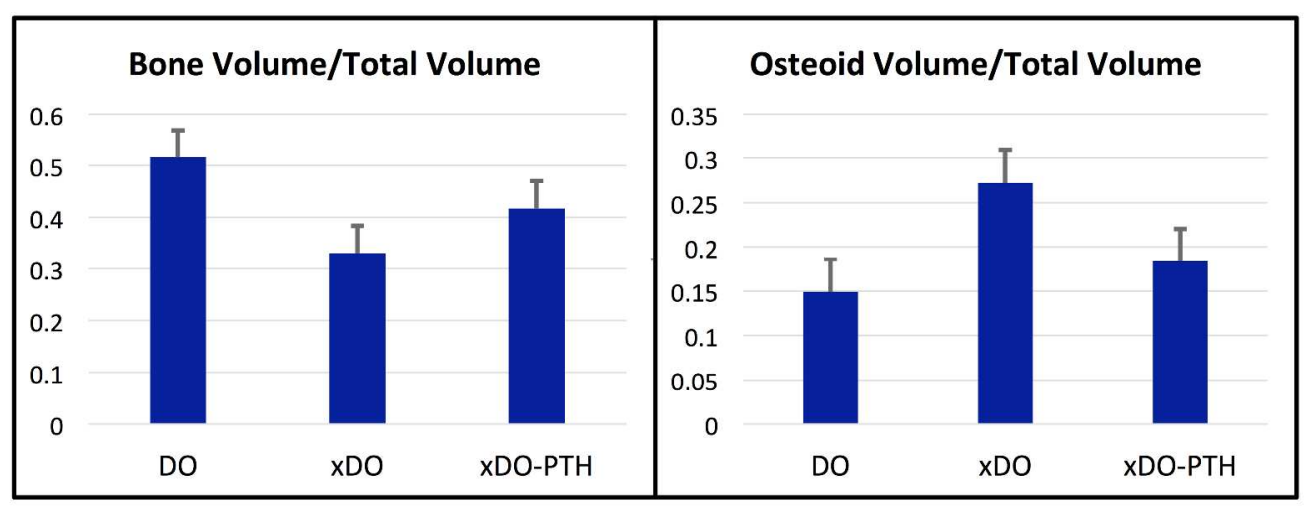

Figure 7. Quantitative histomorphometric analysis. Compared to the non-radiated DO group, radiation treatment in the xDO group resulted in a significant decrease in bone-volume-to-total-volume ratio (BV/TV) $(p=0.008)$. Treatment of irradiated bone with PTH resulted in an increase in BV/TV, but this difference was not significant $(p=0.420)$. Radiation treatment resulted in a significantly greater osteoid-volume-to-total-

volume (OV/TV) in the XDO group, compared to the non-radiated DO group $(p=0.024)$. Treatment of

irradiated bone with PTH resulted in a decrease in OV/TV, but this difference was not significant $(p=0.178)$. However, there was no detectable difference in OV/TV between the non-radiated DO group and the irradiated group treated with PTH $(p=0.345)$.

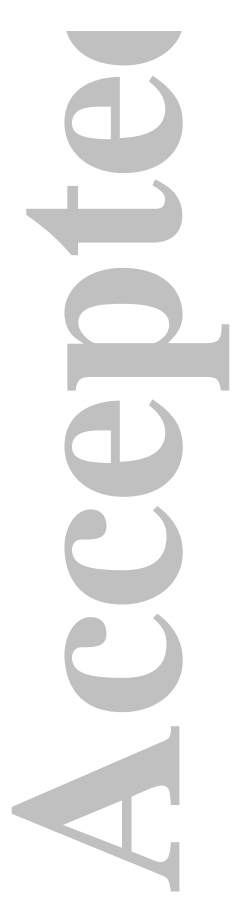

$716 \times 273 \mathrm{~mm}(144 \times 144 \mathrm{DPI})$

John Wiley \& Sons, Inc.

This article is protected by copyright. All rights reserved. 